\title{
Stability Analysis of Nonlinear Dynamic Inversion Controller Applied to Winged Rocket
}

\author{
By Hiroshi YAmasaKI, ${ }^{1)}$ Koichi Yonemoto, ${ }^{1)}$ Kyoshiro ItakURA, ${ }^{1)}$ Guna Surendra Gossamsetti, ${ }^{1}$ \\ Masatomo ICHIGE $^{1)}$ and Yusuke URA ${ }^{1)}$ \\ ${ }^{1)}$ Kyushu Institute of Technology, Kitakyushu, Fukuoka, Japan
}

(Received July 29th, 2015)

\begin{abstract}
Space transportation systems have recently become an active area of development all over the world. Future reusable space transportation systems must possess good stability performance to compensate for the variations in flight conditions. One of the key technologies used for the development of a high performance controller for nonlinear dynamics systems is a dynamic inversion that transforms a nonlinear system into a linear one with pseudo inputs and outputs. It is quite an effective method to control nonlinear systems, and justifies the reason why many advanced aerospace vehicles employ a dynamic inversion controller. In this paper, vector analysis, state trajectory, Lyapunov exponent, and time series analyses results of the Proportional-Derivative (PD) and Dynamic Inversion (DI) control laws will be compared.
\end{abstract}

Key Words: Stability Analysis, Nonlinear System, Autonomous, Dynamic Inversion, Winged Rocket

Nomenclature

$\begin{array}{lll}V & : \text { velocity } & {[\mathrm{m} / \mathrm{s}]} \\ \alpha & : \text { angle of attack } & {[\mathrm{rad}]} \\ \theta & : \text { pitch angle } & {\left[{ }^{\circ}\right]} \\ Q & : \text { pitching angular velocities } & {[\mathrm{rad} / \mathrm{s}]} \\ m & : \text { vehicle mass } & {[\mathrm{kg}]} \\ \rho & : \text { air density } & {\left[\mathrm{kg} / \mathrm{m}^{3}\right]} \\ g & : \text { gravitational acceleration } & {\left[\mathrm{m} / \mathrm{s}^{2}\right]} \\ S & : \text { wing area } & {\left[\mathrm{m}^{2}\right]} \\ b & : \text { wing span } & {[\mathrm{m}]} \\ \bar{c} & : \text { mean aerodynamic chord } & {[\mathrm{m}]} \\ I_{y y} & : \text { moment of inertia around } \mathrm{Y} & \\ \delta_{e} & : \text { axis } & {\left[\mathrm{kg} \cdot \mathrm{m}^{2}\right]} \\ \delta_{e_{r \text { rim }}} & : \text { trim elevator angle } & {\left[{ }^{\circ}\right]} \\ C_{i} & : \text { aerodynamic coefficient } & {\left[{ }^{\circ}\right]}\end{array}$

Subscripts

\begin{tabular}{|c|c|c|}
\hline$L, D$ & : lift, drag & {$[-]$} \\
\hline$m$ & : pitching moment & {$[-]$} \\
\hline$n_{\alpha}$ & $\begin{array}{l}\text { stability derivatives of angle } \\
\text { of attack }\end{array}$ & {$\left[/^{\circ}\right]$} \\
\hline$m_{S}$ & : elevator effectiveness & {$\left[/^{\circ}\right]$} \\
\hline$l_{\text {q }}$ & $\begin{array}{l}\text { stability derivatives of } \\
\text { pitching angular velocity }\end{array}$ & {$[/ \mathrm{rad}]$} \\
\hline$n$ & $\begin{array}{l}\text { : } \text { stability derivatives of attack } \\
\text { angular velocity }\end{array}$ & {$[/ \mathrm{rad}]$} \\
\hline
\end{tabular}

\section{Introduction}

Many researchers have been studying the dynamic inversion (DI) theory and its application to nonlinear systems. Brinker et al. evaluated the robustness of the DI control law for aircrafts. ${ }^{1)}$ Ochi and Kanai studied flight control systems using feedback linearization. ${ }^{2)}$ However, it is very difficult the stability analysis of a nonlinear system. Therefore, most conventional research evaluates flight simulation results or linear stability analyses with a short-period model based on linearized motion equations. ${ }^{3,4)}$ The flight simulation results are meaningful, but they cannot ensure rigorous stability. Since very little research has focused on the stability of DI, it is important to study the stability of the DI control law on a nonlinear system.

The DI theory has an observed, characteristic quantity, and a control quantity that are pseudo-linearized. Therefore, it is possible to express the state diagram of observables by vector analysis. Since vector analysis represents a state transition in a graphical manner, it is easy to understand the effectiveness of the DI theory. However, it is difficult to visualize the status of more than three dimensions. A vector analysis is limited to the visual representation of variables. One of the most famous stability analysis of nonlinear controller is the Lyapunov function. ${ }^{5,6)}$ Stability is evaluated by examining the rate of change of the potential energy around the equilibrium point. However, the Lyapunov stability analysis needs a defined Lyapunov function in an area near the equilibrium point. Therefore, it is difficult to utilize the Lyapunov method for the aircraft whose equilibrium point changes. In addition, if the system is locally stable, local linear analysis can be employed without the use of a Lyapunov function. ${ }^{7-9)}$ Therefore, a Lyapunov exponent index is needed to assess the global stability of the function. In a dynamic system, the spectrum of Lyapunov exponents plays a very important role in the determination on whether a system is unstable or not. Any 
system containing at least one positive Lyapunov exponent is defined to be unstable. ${ }^{10)}$ One important property is that the sum of Lyapunov exponents is related to the generalized divergence of the flow of the system. In a mechanical system, damping is related to the energy dissipation. Thus, the sum of Lyapunov exponents must be related to the damping of a mechanical system and can be utilized to monitor any damping changes in the system.

This paper addresses this stability analysis method and its results, and discusses its applicability to a winged rocket. Section 2 is the introduction of the winged rocket model and its dynamics. Section 3 is the explanation of the simple DI theory. In Section 4, the method of stability analysis is explained, including the transfer function and state equation. The authors also discuss the basic characteristics of the DI theory along with an explanation of the Lyapunov exponents. Section 5 reports the results of the stability analyses (vector, trajectory, time series, Lyapunov exponent analysis) performed based on the PD control law and the DI control law. Finally, the authors summarize their conclusions and plans for future works.

\section{Winged Rocket Dynamics}

\subsection{Introduction of winged rocket model}

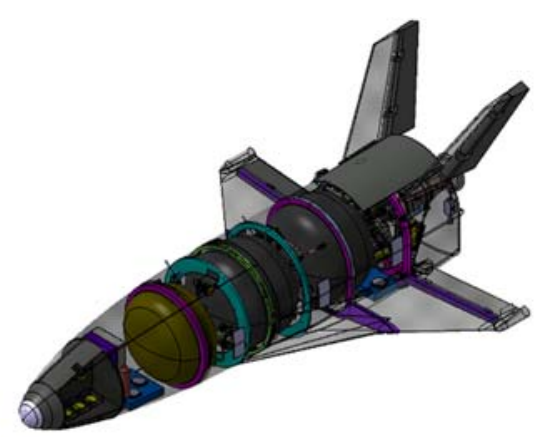

Fig. 1. Subscale winged rocket WIRES\#015.

Table 1. Specification of WIRES\#015.

\begin{tabular}{|l|l|l|}
\hline Total Mass & {$[\mathrm{kg}]$} & 500 \\
\hline Total Length & {$[\mathrm{m}]$} & 4 \\
\hline Body Diameter & {$[\mathrm{m}]$} & 0.76 \\
\hline Wing Area & {$\left[\mathrm{m}^{2}\right]$} & 2.07 \\
\hline Wing Span & {$[\mathrm{m}]$} & 2.51 \\
\hline Mean Aerodynamic Chord & {$[\mathrm{m}]$} & 0.938 \\
\hline Center of Gravity & {$[\%]$} & 65 \\
\hline
\end{tabular}

The authors are developing a subscale, experimental winged rocket $^{11,12)}$ called the WInged REusable Sounding rocket \#015 (WIRES\#015) whose objective is the demonstration of an operational navigation, guidance and control (NGC) system, for the vehicle using elevons and rudders to control its attitude. Figure 1 shows the overview of WIRES\#015. The aerodynamic design of the structure employs the shape of the HIghly Maneuverable Experimental Space vehicle (HIMES) that was designed by the Japan Aerospace Exploration Agency/the Institute of Space and Astronautical Science (JAXA/ISAS) in the 1980's. Table 1 shows the specification of WIRES\#015.

\subsection{Dynamics of winged rocket}

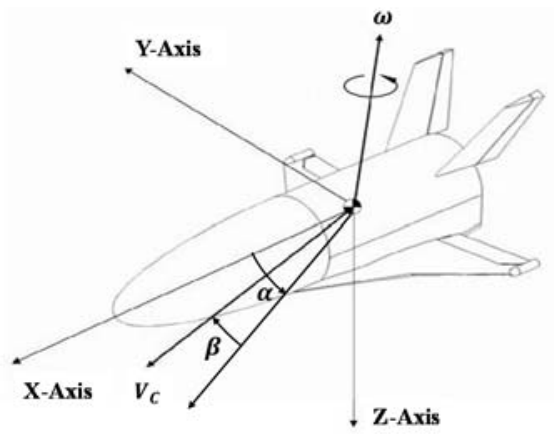

Fig. 2. Winged rocket body coordinates.

In this section, the dynamics of the winged rocket are explained. First, the winged rocket body coordinates are set as shown in Fig. 2. The winged rocket has nonlinear dynamics when in a flight environment, and the rocket longitudinal dynamics of the model are thus expressed by Eqs. (1)-(6).

$$
\begin{aligned}
& \dot{\mathbf{x}}=f(\mathbf{x})+g(\mathbf{x}) u \\
& \mathbf{x}=\left[\begin{array}{llll}
V & \alpha & Q & \theta
\end{array}\right]^{T} \\
& f(\mathbf{x})=\left[\begin{array}{llll}
f_{1}(\mathbf{x}) & f_{2}(\mathbf{x}) & f_{3}(\mathbf{x}) & f_{4}(\mathbf{x})
\end{array}\right]^{T} \\
& g(\mathbf{x})=\left[\begin{array}{llll}
0 & 0 & g_{3}(\mathbf{x}) & 0
\end{array}\right]^{T} \\
& u=\left[\delta_{e}\right] \\
& f_{1}(\mathbf{x})=-g \sin (\theta-\alpha)-\frac{\rho V^{2} S}{2 m} C_{D} \\
& f_{2}(\mathbf{x})=Q+\frac{g}{V} \cos (\theta-\alpha)-\frac{\rho V S}{2 m} C_{L} \\
& f_{3}(\mathbf{x})=\frac{\rho V^{2} \overline{S c}}{2 I_{y y}}\left(C_{m}+\frac{\bar{c}}{2 V}\left(C_{m_{q}} Q+C_{m_{\dot{\alpha}}} \dot{\alpha}\right)\right) \\
& f_{4}(\mathbf{x})=Q \\
& g_{3}(\mathbf{x})=\frac{\rho V^{2} S \bar{c}}{2 I_{y y}} C_{m_{\delta_{e}}}
\end{aligned}
$$

where $\mathbf{x}$ is the state vector of the longitudinal, and Eq. (1) expresses a nonlinear affine system.

\section{Dynamic Inversion}

Dynamic inversion is a well-established branch of study in control theory that performs a linearization of the input and output variables by the $n$ number of derivatives with respect to the observables in the DI theory. Therefore, it is possible to pseudo-linearize nonlinear state equations.

\subsection{Dynamic inversion of SISO system}

Nonlinear dynamics are generally expressed by the following equations [Eqs. (7) and (8)]:

$$
\begin{gathered}
\dot{\mathbf{x}}(t)=f(\mathbf{x}(t))+g(\mathbf{x}(t)) u(t) \\
y(t)=h(\mathbf{x}(t))
\end{gathered}
$$


Assuming that the system has relative degree $n$, and the new state $z$ is defined as follows:

$$
L_{f} h=\frac{d h}{d x} f=\frac{\partial h}{\partial x_{1}} f_{1}+\frac{\partial h}{\partial x_{2}} f_{2}+\cdots+\frac{\partial h}{\partial x_{n}} f_{n}
$$

where $L_{f}$ is the Lie derivative. For the functions $h(\mathbf{x}) \in R$, $f(\mathbf{x})=\left[\begin{array}{llll}f_{1}(\mathbf{x}) & f_{2}(\mathbf{x}) & \cdots & f_{n}(\mathbf{x})\end{array}\right]^{T}$ and $g(\mathbf{x}) \in R^{n}$, it follows that if $x \in R^{n}$, and a recursive use is applied with $L^{0}{ }_{f}=h$, that

$$
L_{g} L_{f} h=\frac{\partial L_{f} h}{\partial x_{i}} g_{i}+\frac{\partial L_{f} h}{\partial x_{i}} g_{i}+\cdots+\frac{\partial L_{f} h}{\partial x_{i}} g_{i} \neq 0
$$

where $L^{i+1} h$ is defined as,

$$
L^{i+1} h=L_{f}\left(L_{f}^{i} h\right), i=1,2, \ldots
$$

whereby $x$ is omitted.

If there is an input command $u$ and the observer quantity $y$ is the $n^{\text {th }}$ derivative, the feedback control law can be expressed as follows:

$$
u=\frac{1}{L_{g} L^{n-1}{ }_{f} h}\left(v-L_{f}^{n} h\right)
$$

where $v$ is the pseudo input in this system. The designed property and the stability control law stabilize the observer variables.

\subsection{Dynamic inversion of the aircraft}

Longitudinal aerodynamic models can be applied to the Single-input Single-output (SISO) system, so the control input is expressed as follows. The observer of the longitudinal model is defined in terms of the angle of attack [Eq. (12)]. Additionally, Eqs. (13)-(15) are intermediate expressions that can be derived from Eqs. (12) and (16).

$$
\begin{gathered}
\alpha=h(\mathbf{x}) \\
\ddot{\alpha}=L_{f}^{2} h(\mathbf{x})+L_{g} L_{f} h(\mathbf{x}) \\
\ddot{\alpha}=\frac{\partial \dot{\alpha}}{\partial V} \dot{V}+\frac{\partial \dot{\alpha}}{\partial \alpha} \dot{\alpha}+\frac{\partial \dot{\alpha}}{\partial Q} \dot{Q}+\frac{\partial \dot{\alpha}}{\partial \theta} \dot{\theta} \\
\ddot{\alpha}=\sum_{i=1}^{4} J_{2 i}(\mathbf{x}) f_{i}(\mathbf{x})+J_{23}(\mathbf{x}) g_{3}(\mathbf{x}) \delta_{e} \\
\delta_{e}=\frac{1}{J_{23}(\mathbf{x}) g_{3}(\mathbf{x})}\left(\ddot{\alpha}-\sum_{i=1}^{4} J_{2 i}(\mathbf{x}) f_{i}(\mathbf{x})\right)
\end{gathered}
$$

where $J(\mathbf{x})$ is the Jacobian of the model dynamics.

$$
\ddot{\mathbf{x}}=J(\mathbf{x}) \dot{\mathbf{x}}
$$

Herein, the elevator angle represents the control inputs, and the pseudo input is the second derivative of the angle of attack. Therefore, the relation of the elevator angle and the pseud input are expressed the following equation [Eq. (17)].

$$
\delta_{e}=\frac{1}{J_{23}(\mathbf{x}) g_{3}(\mathbf{x})}\left(v-\sum_{i=1}^{4} J_{2 i}(\mathbf{x}) f_{i}(\mathbf{x})\right)
$$

\section{Stability Analysis}

\subsection{Transfer function}

Pseudo inputs are designed by a stable PD control law given by Eq. (18),

$$
v=\ddot{\alpha}=K_{\alpha}\left(\alpha_{c o m}-\alpha\right)-K_{\dot{\alpha}} \dot{\alpha}
$$

and the transfer functions for the angle of attack is,

$$
\frac{\alpha}{\alpha_{c o m}}=\frac{K_{\alpha}}{s^{2}+K_{\dot{\alpha}} s+K_{\alpha}}
$$

The gain was designated as $K_{\alpha}=2.0$ and $K_{\dot{\alpha}}=2.5$. Therefore, in the case of the DI theory, the natural frequency of the angle of attack becomes equal to 1.414 and the damping ratio becomes equal to 0.884 .

\subsection{State equation}

Observation quantities converge when the pseudo inputs are stable. However, in the case of a nonlinear system, it is difficult to represent the entire system with a transfer function. The feedback control law of DI is expressed using the Jacobian component expressed by the following equation:

$$
\begin{gathered}
\dot{\mathbf{x}}=M(\mathbf{x}) f(\mathbf{x})+G\left(\mathbf{x}, \alpha_{\text {com }}\right) \\
M(\mathbf{x})=\left[\begin{array}{cccc}
1 & 0 & 0 & 0 \\
0 & 1 & 0 & 0 \\
-J_{21} / J_{23} & -\left(K_{\dot{\alpha}}+J_{22}\right) / J_{23} & 0 & -J_{24} / J_{23} \\
0 & 0 & 0 & 1
\end{array}\right] \\
G\left(\mathbf{x}, \alpha_{\text {com }}\right)=\left[\begin{array}{c}
0 \\
0 \\
K_{\alpha}\left(\alpha_{c o m}-\alpha\right) / J_{23} \\
0
\end{array}\right]
\end{gathered}
$$

The purpose of the DI is to pseudo-linearize the relationship between the control inputs and control outputs. Therefore, the dynamic characteristics of the aircraft do not impose any longer an effect in relation to this formula. However, state equations using the DI theory have nonlinear terms, so the state quantities (other than the controlled object) are also nonlinear. Therefore, it is necessary to analyze their motions.

\subsection{Lyapunov exponents}

The continuous time, $\mathrm{n}$-dimensional, autonomous, dynamic system is given by $\boldsymbol{x}\left(t_{0}\right)=\boldsymbol{x}, \boldsymbol{x}(t) \in \mathrm{R}^{\mathrm{n}}$

$$
\dot{\mathbf{x}}(t)=f\left(x_{1}, x_{2}, \cdots, x_{n}\right)=\left[\begin{array}{llll}
\frac{d x_{1}}{d t} & \frac{d x_{2}}{d t} & \cdots & \frac{d x_{n}}{d t}
\end{array}\right]^{T}
$$

Eq. (23) determines the set of solution curves in phase space. The $i^{\text {th }}$ Lyapunov exponent in the n-dimensional phase space is defined in terms of the length of the $i^{\text {th }}$ principal axis $P_{i}(t)$ of the ellipsoid. ${ }^{13)}$ Herein, $P_{i}(t)$ is $\dot{x}(t)$ 


$$
\lambda_{i}=\lim _{t \rightarrow \infty} \frac{1}{t} \ln \frac{P_{i}(t)}{P_{i}(0)}
$$

where $\lambda_{i}$ ranges from the largest to the smallest value. The Lyapunov exponents defined in Eq. (24) are not very helpful for the computation because it is impossible to average infinitely. Thus, the Lyapunov exponent may be estimated, and becomes a function of time or a function of the number of iterations.

\section{Stability Analysis Results}

Herein, the authors present the results of the vector analysis, Liapunov analysis, state trajectory, and time response analyses. Moreover, the authors used the PD control law in order to qualitatively compare the results. The PD control law is expressed the following equation [Eq. (25)].

$$
\begin{aligned}
& \delta_{e}=K_{\alpha}\left(\alpha_{\text {com }}-\alpha\right) 180 / \pi-\left(K_{\dot{\alpha}} \dot{\alpha}\right) 180 / \pi+\delta_{e_{\text {trim }}} \\
& K_{\alpha}=-2.0 \quad K_{\dot{\alpha}}=-2.5 \quad \delta_{e_{\text {rrim }}}=-4.32
\end{aligned}
$$

where $K_{\alpha}$ is gain of angle of attack, $K_{\dot{\alpha}}$ is gain of attack angular velocity, and $\delta_{e_{\text {trim }}}$ is trim of elevator. The authors confirmed that it is stable by the linearization system, because the gain is sufficient for a qualitative assessment. $\alpha_{c o m}$ is the reference of the angle of attack.

\subsection{Vector analysis}

Vector analysis is a method of visually analyzing a state transition. Using vector analysis, the effectiveness of the DI theory becomes evident. However, it is necessary to perform a complex vector analysis due to nonlinear rocket dynamics. However, if it utilizes the DI theory for a nonlinear system, it is possible to analyze the observed quantity. Figure 3 shows the vector diagram of the angle of attack by a DI control system. In accordance to Fig. 3, it can be deduced that the DI theory converges to the target value (target is $12^{\circ} \approx 0.2094$ $\mathrm{rad})$.

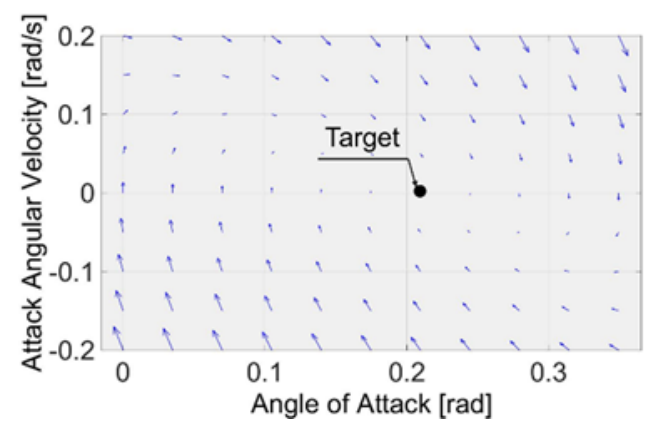

Fig. 3. Vector of observed quantity by the DI control system.

\subsection{Lyapunov analysis}

Figures 4-11 show the Lyapunov exponents of various parameters using the PD and DI control laws. Any system containing at least one positive Lyapunov exponent is defined to be unstable.

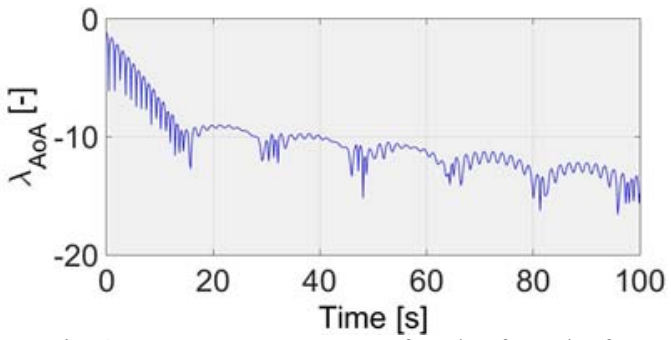

Fig. 4. Lyapunov exponents of angle of attack of PD.

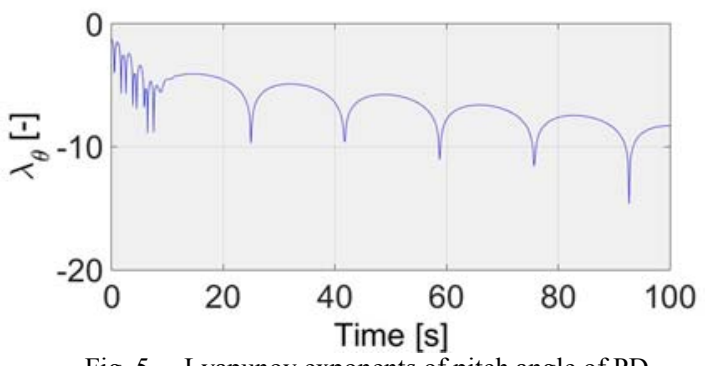

Fig. 5. Lyapunov exponents of pitch angle of PD.

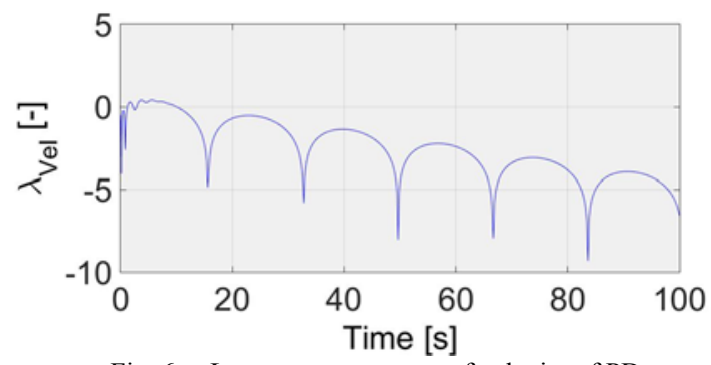

Fig. 6. Lyapunov exponents of velocity of PD.

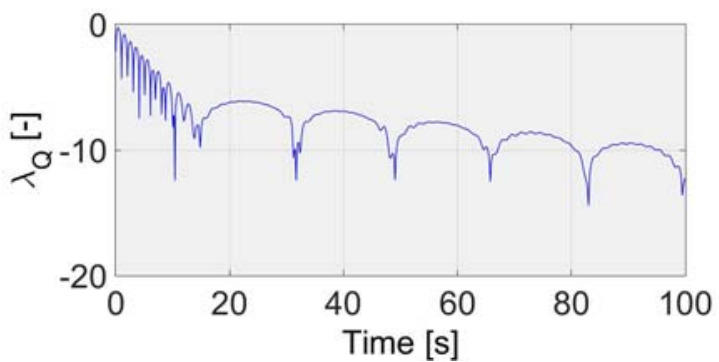

Fig. 7. Lyapunov exponents of pitching angular velocity of PD.

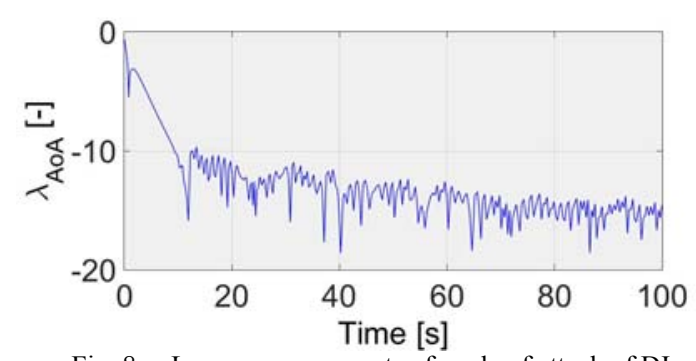

Fig. 8. Lyapunov exponents of angle of attack of DI.

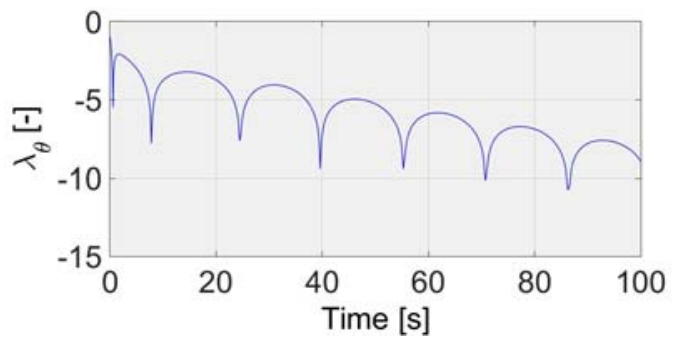

Fig. 9. Lyapunov exponents of pitch angle of DI. 


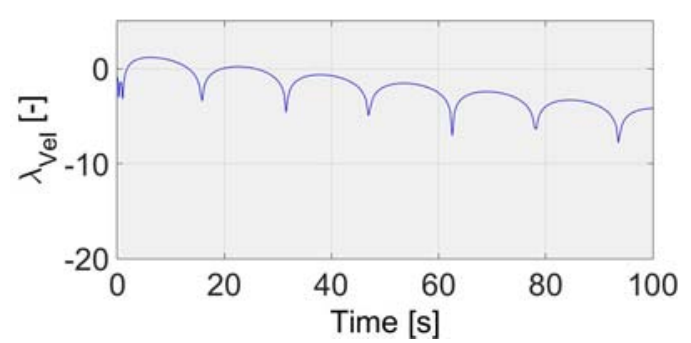

Fig. 10. Lyapunov exponents of velocity of DI.

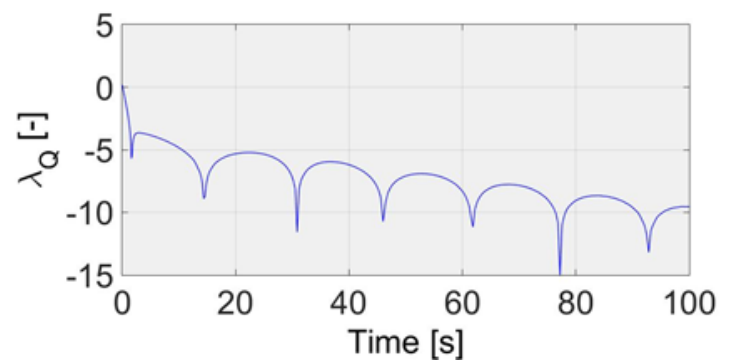

Fig. 11. Lyapunov exponents of pitching angular velocity of DI.

Based on these results, it can be deduced that this system is stable.

\subsection{State trajectory}

For a more thorough analysis, the state trajectory elicited a random value for the initial point, yet still converged to the equilibrium point. State trajectories can result in converging orbits of some parameters. For this reason the authors confirmed the trajectory of angle of attack, pitch angle, and velocity. State ranges listed randomly are shown in Table 2.

Table 2. State ranges.

\begin{tabular}{|l|l|}
\hline $30 \leq V_{0} \leq 150$ & {$[\mathrm{~m} / \mathrm{s}]$} \\
\hline $0 \leq \alpha_{0} \leq 20$ & {$\left[{ }^{\circ}\right]$} \\
\hline$-1 \leq Q_{0} \leq 1$ & {$[\mathrm{rad} / \mathrm{s}]$} \\
\hline$-60 \leq \theta_{0} \leq 30$ & {$\left[^{\circ}\right]$} \\
\hline
\end{tabular}

Figure 12 shows the state trajectory of the PD control law, and Figs. 13-16 show the state of phase plots for various parameters using PD control. In addition, Fig. 17 shows the state trajectory of the DI control law, and Figs. 18-21 show the state of phase plots for various parameters using DI control. The results converge to the equilibrium point, indicating a stable system.

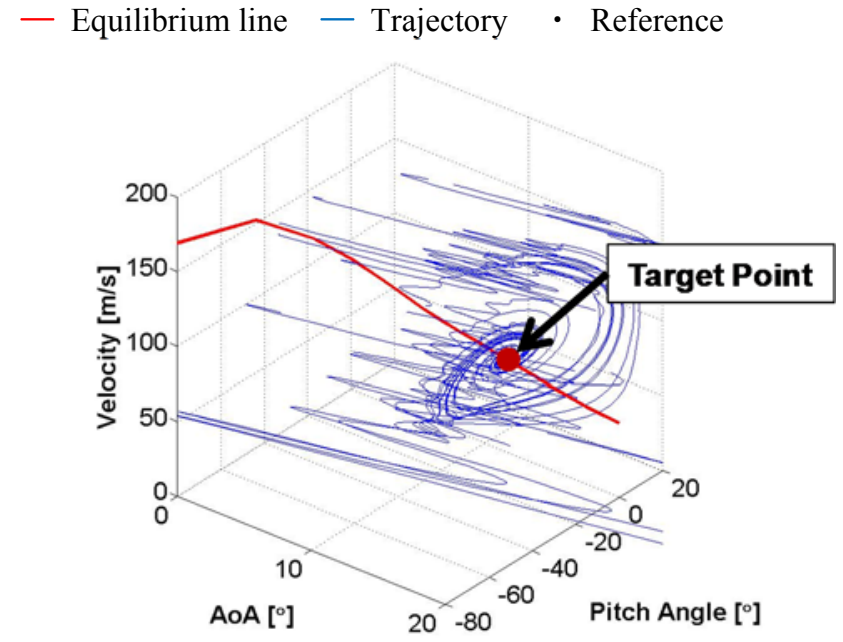

Fig. 12. State trajectory of PD control.

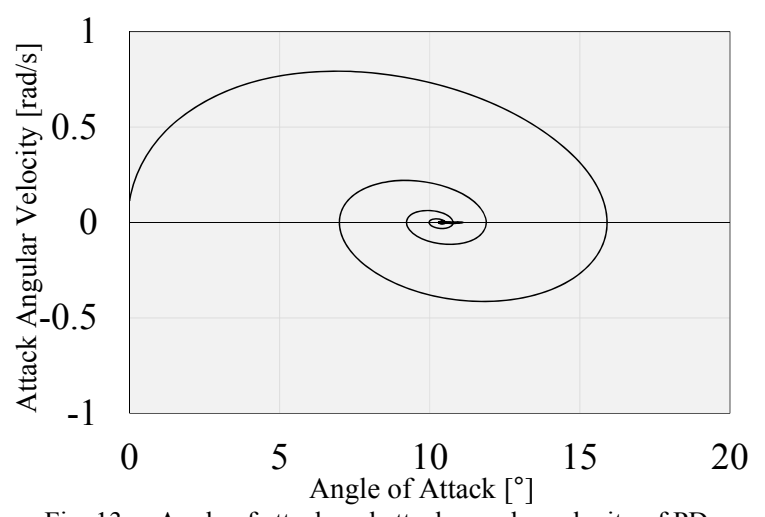

Fig. 13. Angle of attack and attack angular velocity of PD.

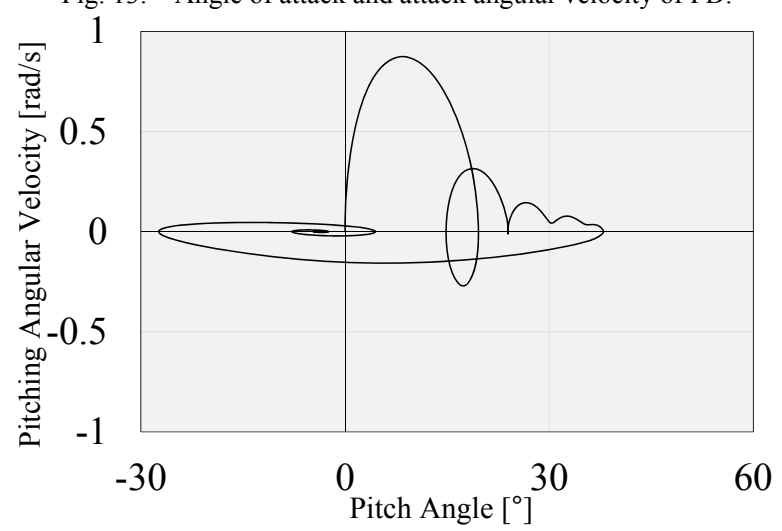

Fig. 14. Pitch angle and pitching angular velocity of PD.

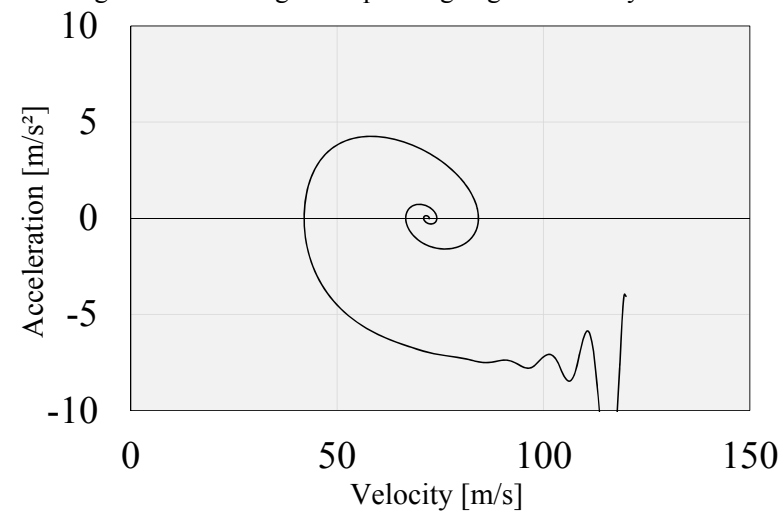

Fig. 15. Velocity and acceleration of velocity of PD. 


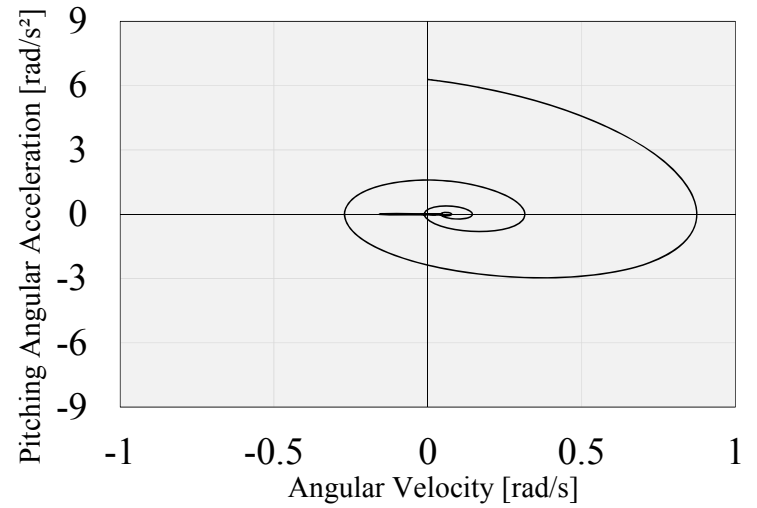

Fig. 16. Pitching angular velocity and pitching angular acceleration of $\mathrm{PD}$

\section{— Equilibrium line - Trajectory $\quad$ Reference}

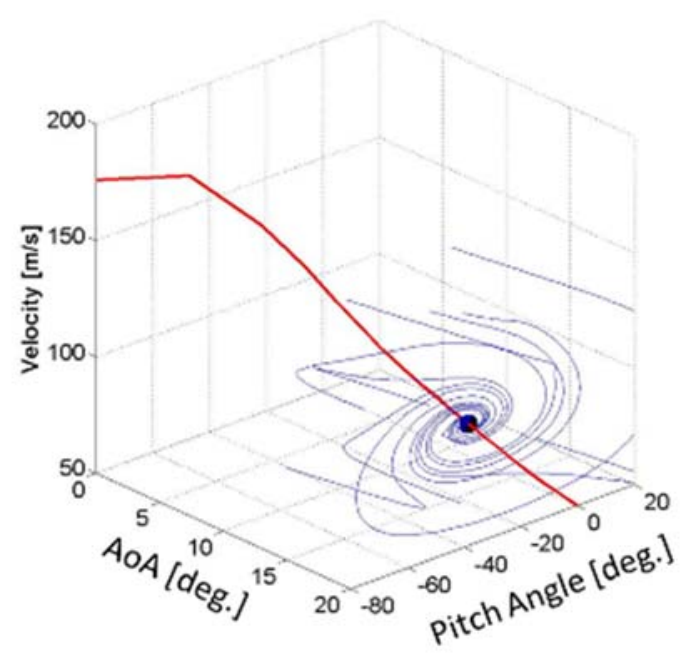

Fig. 17. State trajectory of DI control.

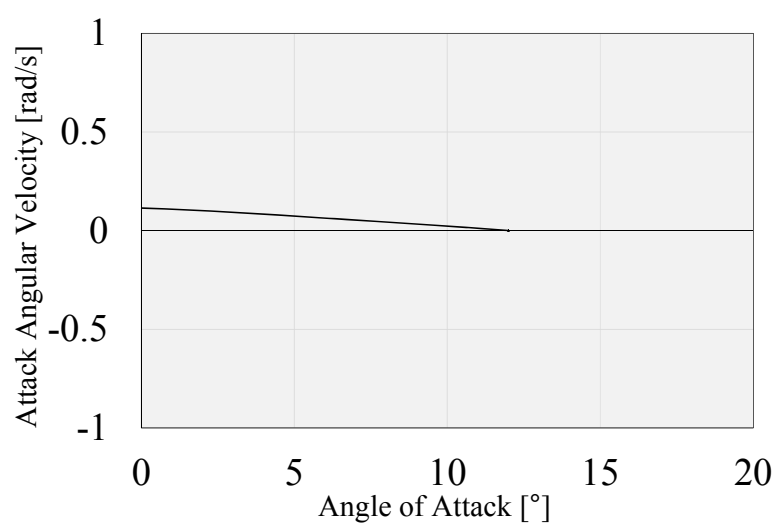

Fig. 18. Angle of attack and attack angular velocity of DI.

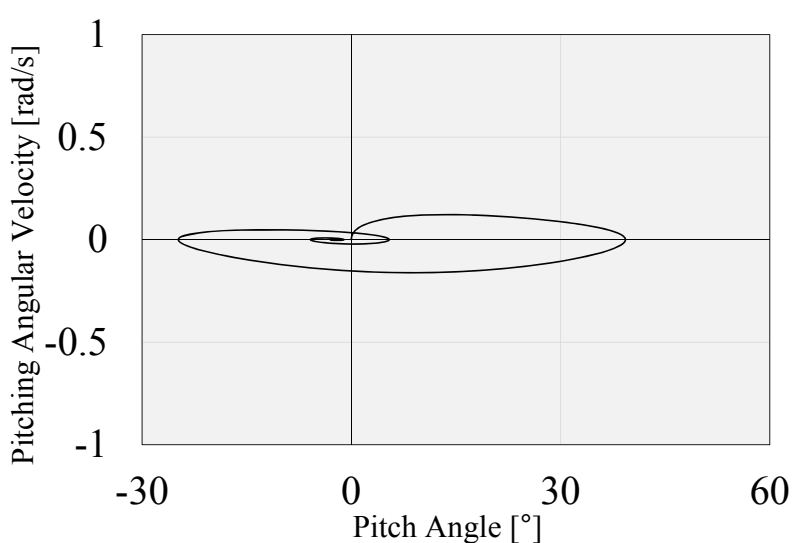

Fig. 19. Pitch angle and pitching angular velocity of DI.

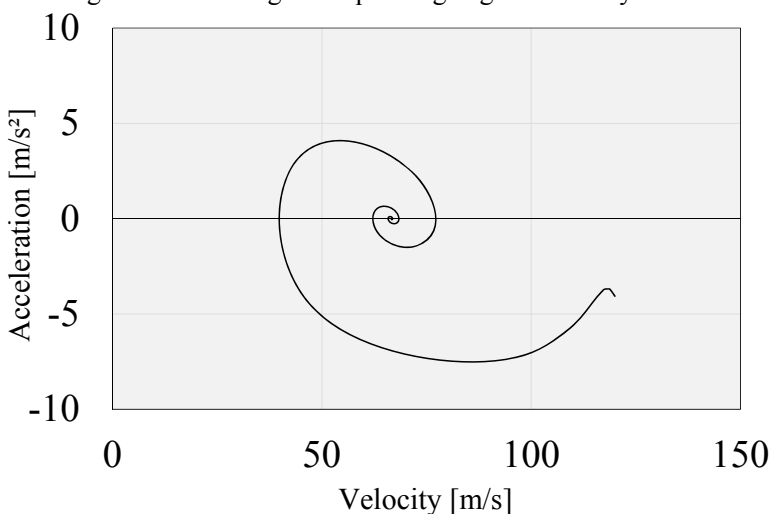

Fig. 20. Velocity and acceleration of velocity of DI.

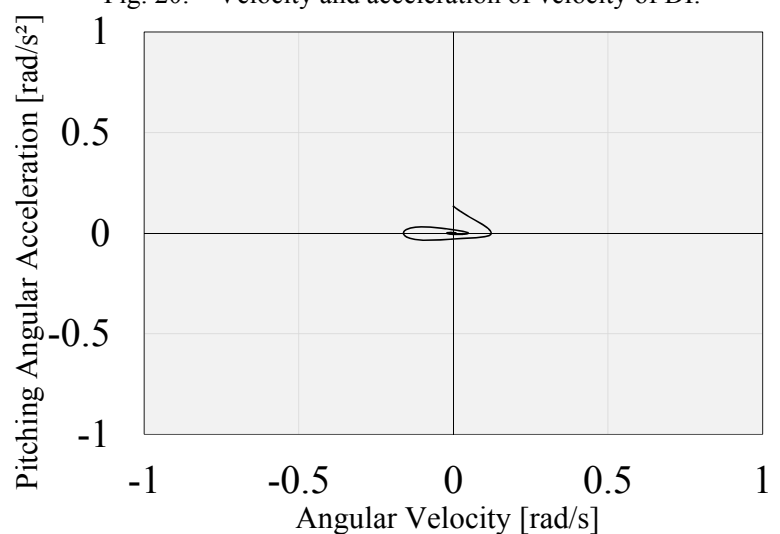

Fig. 21. Pitching angular velocity and pitching angular acceleration of DI.

\subsection{Time response}

Figures 22-26, and Figs. 27-31 show the time series analyses of the PD and DI control laws. These results indicate a temporal convergence. Figure 18 shows the elevator command of PD. The result of Fig. 23 plays an important role in order to evaluate whether it is a realistically possible control command. Table 3 shows the initial state of the time response.

Table 3. Initial state.

\begin{tabular}{|l|l|l|}
\hline Velocity & {$[\mathrm{m} / \mathrm{s}]$} & 120 \\
\hline Angle of Attack & $\left.{ }^{\circ}\right]$ & 0 \\
\hline Angular Velocity & {$[\mathrm{rad} / \mathrm{s}]$} & 0 \\
\hline Pitch Angle & {$\left[^{\circ}\right]$} & 0 \\
\hline
\end{tabular}




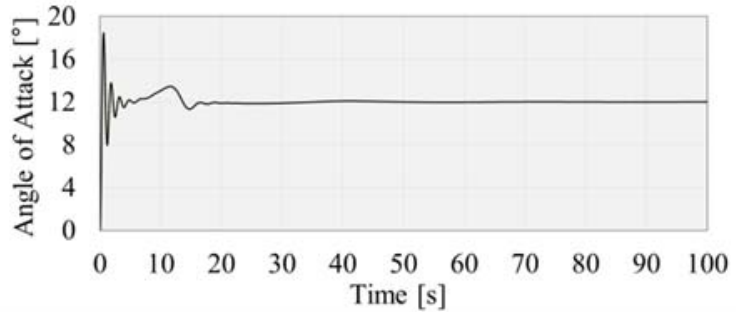

Fig. 22. Time series analysis of angle of attack of PD.

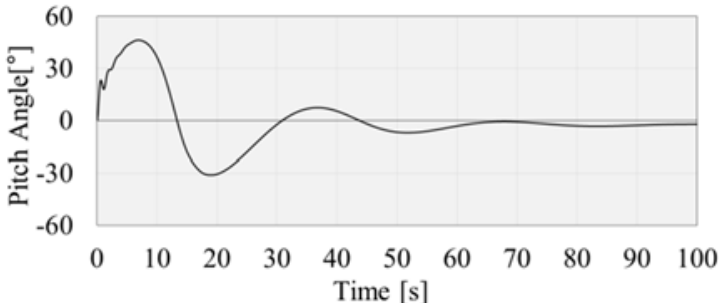

Fig. 23. Time series analysis of pitch angle of PD.

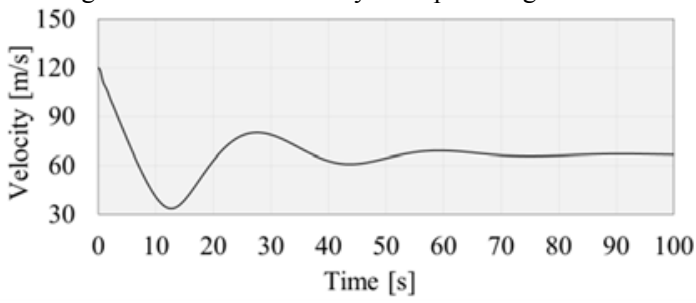

Fig. 24. Time series analysis of velocity of PD.

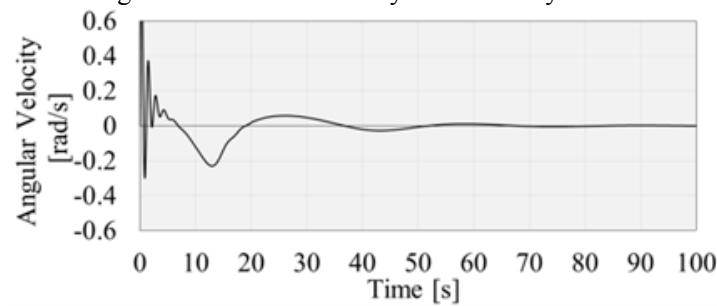

Fig. 25. Time series analysis of angular velocity of PD.

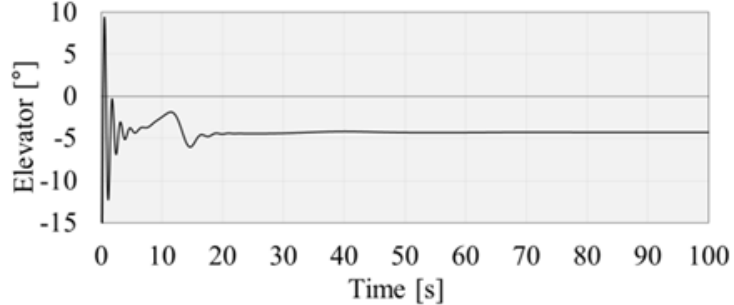

Fig. 26. Time series analysis of elevator command of PD

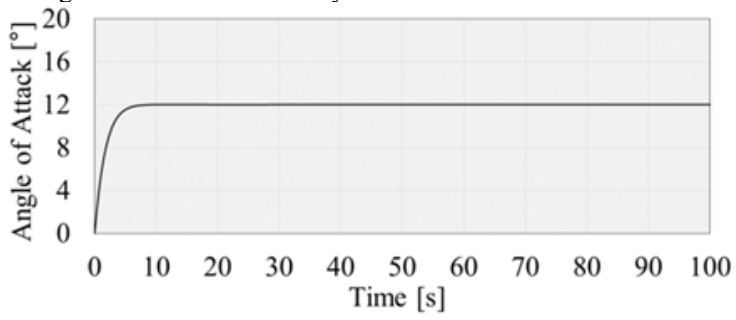

Fig. 27. Time series analysis of angle of attack of DI.

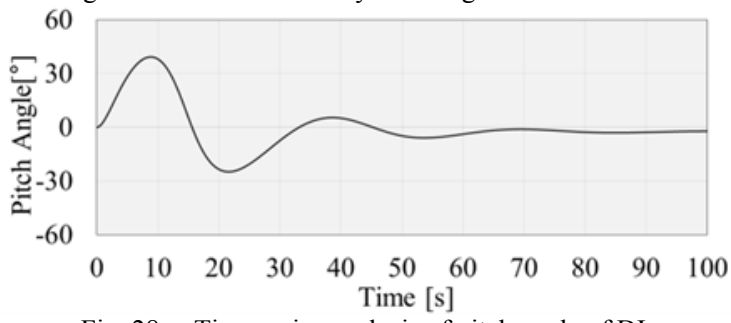

Fig. 28. Time series analysis of pitch angle of DI.

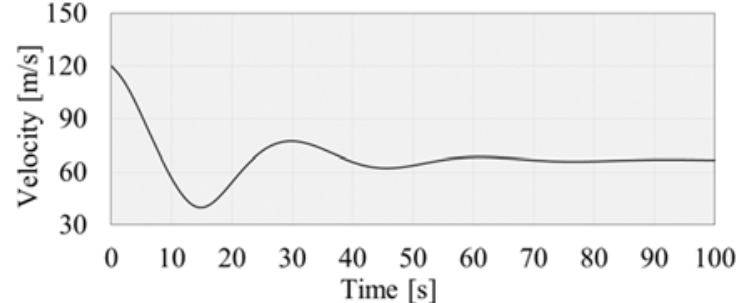

Fig. 29. Time series analysis of velocity of DI.

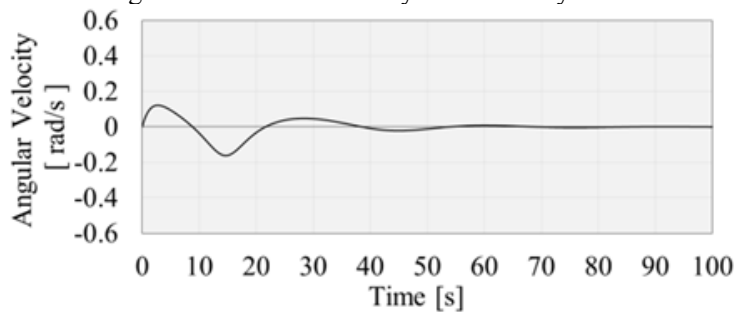

Fig. 30. Time series analysis of angular velocity of DI

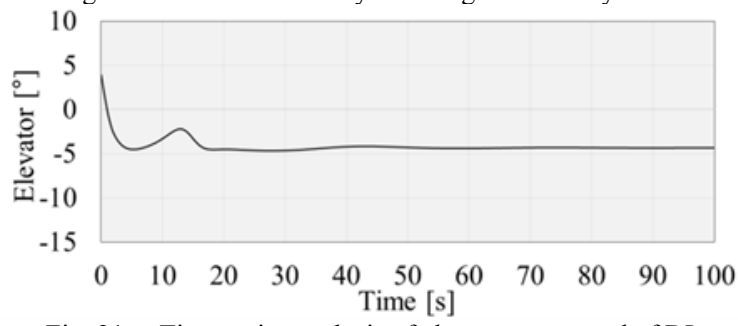

Fig. 31. Time series analysis of elevator command of DI.

\subsection{Discussions}

The following results represent the difference between the PD and DI control laws of nonlinear systems. Comparing Fig. 12 of the PD control law and Fig. 17 of the DI control law, the PD control law displays complex vibration, but the DI control law converges with minimal vibration. This observation is justified by the corresponding phase plots. All of the phase plots of the PD control law tend to converge in a spiral. In contrast, the DI control law's angle of attack converges very quickly and becomes constant, whereas the velocity, pitch angle, and pitching angular velocity converge in a spiral briefly before becoming constant. All of the Lyapunov exponents have shown sensitivity with respect to the initial conditions, even though the sum of all Lyapunov exponents is negative, leading to the expectation that all state variables should have converged uniquely. Based on the time response results, the PD control law exhibits a peculiar vibration response. On the other hand, the DI control law only accounts for phugoid motion and a second order-lag response in regard to the angle of attack. Considering the convergence rate over time, the DI control of the angle of attack converges faster than the PD control, even though other variables converge in time in the same manner as in the case of use of the PD control law.

\section{Conclusion}

Based on the analyses results, both the PD control law and the DI control laws have been shown to be stable in their finite states (Table 2). The DI control law was found to be better for use in a dedicated system controlling the angle of attack since convergence is fast. The PD control law, however, established 
equilibrium in all states using a spiral pattern.

Based on these results, the authors are currently researching the mechanism of convergence. In addition, the authors are exploring ways of improving the DI control system for an adaptive reference model.

\section{References}

1) Brinker, J. S. and Wise, K. A.: Stability and Flying Qualities Robustness of a Dynamic Inversion Aircraft Control Law, Journal of Guidance, Control, and Dynamics, 19 (1996), pp. 1270-1277.

2) Ochi, Y. and Kanai, K.: Design of Restructurable Flight Control Systems Using Feedback Linearization, Journal of Guidance, Control, and Dynamics, 14 (1991), pp. 903-911.

3) Ito, D., et al.: Reentry Vehicle Flight Controls Design Guidelines: Dynamic Inversion. Technical Report, NASA/TP-2002-210771, (2002).

4) Shimozawa, T. and Sagara, S.: Digital Adaptive Control of a Winged Rocket in Wide Range Flight Conditions, In: Modelling, Identification and Control (ICMIC), The 2010 International Conference on IEEE, (2010), pp. 197-202.

5) Branicky, M. S.: Multiple Lyapunov Functions and Other Analysis Tools for Switched and Hybrid Systems. IEEE Transactions on Automatic Control, 43 (1998), pp. 475-482.

6) Feron, E., Apkarian, P. and Gahinet, P.: Analysis and Synthesis of Robust Control Systems via Parameter-dependent Lyapunov Functions. IEEE Transactions on Automatic Control, 41(1996), pp. 1041-1046.
7) Yoden, S. and Nomura, M.: Finite-time Lyapunov Stability Analysis and its Application to Atmospheric Predictability, Journal of the Atmospheric Sciences, 50(1993), pp. 1531-1543.

8) Shin, K. and Hammond, J. K.: The Instantaneous Lyapunov Exponent and its Application to Chaotic Dynamical Systems, Journal of Sound and Vibration, 218 (1998), pp. 389-403.

9) Totoki, Y., Goto, A., Suemitsu, H. and Matuo, T.: Synchronization Detection of Biological CAM Plants Using Instantaneous Lyapunov Exponent, Proc. of SICE Annual Conference, (2010), pp. 514-519.

10) Moon, F. C.: Chaotic Vibrations: An Introduction for Applied Scientists and Engineers, Research supported by NSF, USAF, US Navy, US Army, and IBM. New York, Wiley-Interscience, 1 (1987), pp. 322.

11) Yonemoto, K., et al.: Current Status of Experimental Winged Rocket Development, The Proceedings of 2010 Asia-Pacific International Symposium on Aerospace Technology, Xi'an China, 1, (2010).

12) Itakura, K., et al.: Development and Ground Combustion Test of a Subscale Reusable Winged Rocket, Transactions of the Japan Society for Aeronautical and Space Sciences, Aerospace Technology Japan, 12, ists29 (2014), pp.To_3_1-To_3_5.

13) Glendinning, P. and Sparrow, C.: Local and Blobal Behavior near Homoclinic Orbits, Journal of Statistical Physics, 35 (1984), pp. 645-696. 